\title{
Connective tissue growth factor induces osteogenic differentiation of vascular smooth muscle cells through ERK signaling
}

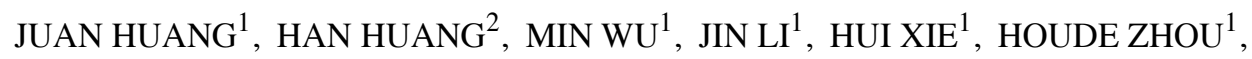 \\ ERYUAN LIAO ${ }^{1}$ and YIQUN PENG ${ }^{1}$ \\ ${ }^{1}$ Institute of Endocrinology and Metabolism, The Second Xiangya Hospital of Central South University, \\ Changsha, Hunan; ${ }^{2}$ Central South University School of Public Health, Changsha, Hunan, P.R. China
}

Received February 13, 2013; Accepted April 11, 2013

DOI: $10.3892 /$ ijmm.2013.1398

\begin{abstract}
Connective tissue growth factor (CTGF) plays an important role in the pathogenesis of atherosclerosis by promoting vascular smooth muscle cell (VSMC) growth, migration, apoptosis, adhesion and the secretion of matrix components. The osteogenic differentiation of VSMCs is essential in the development of vascular calcification. However, the role of CTGF in the transdifferentiation and calcification of VSMCs is unclear. In the present study, we examined whether CTGF stimulates VSMC transdifferentiation. Primary VSMCs were obtained from mouse thoracic aortas by enzymatic digestion and identified by immunostaining for smooth muscle specific $\alpha$-actin antibody ( $\alpha$-SMA). VSMC calcification was induced by the addition of CTGF to the osteogenic mediaum containing 5-10\% FBS in the presence of $0.25 \mathrm{mM}$ ascorbic acid and $10 \mathrm{mM} \beta$-glycerophosphate for 14 days. Calcified cells were determined by Alizarin Red S staining. Our results revealed that CTGF induced the expression of several bone markers, including alkaline phosphatase (ALP), osteocalcin (OC), osteoprotegerin (OPG) and core-binding factor subunit $\alpha 1$ (Cbf $\alpha 1)$ /runt-related transcription factor 2 (Runx2), as well as calcification. However, the inhibition of extracellular signal-regulated kinase (ERK) activity using the ERK-specific inhibitor, PD98059, blocked the induction of these proteins and VSMC calcification. Based on these data, we conclude that CTGF stimulates the transdifferentiation of VSMCs into osteoblasts and that the ERK signaling pathway appears to play a critical role in this process.
\end{abstract}

\section{Introduction}

Vascular calcification is prevalent in patients with atherosclerosis, aging, end-stage renal failure, uremia and type 2 diabetes and is a strong predictor of cardiovascular morbidity and

Correspondence to: Mrs Yiqun Peng, Institute of Endocrinology and Metabolism, The Second Xiangya Hospital of Central South University, 139 Middle Renmin Road, Changsha, Hunan 410011, P.R. China E-mail: yqpeng@126.com

Key words: connective tissue growth factor, vascular calcification, osteoblast, vascular smooth muscle cells, atherosclerosis mortality (1-3). In previous years, vascular calcification was considered to be a passive process. However, vascular calcification is now recognized as an organized, highly regulated process which is similar to mineralization in bone tissue $(4,5)$. A number of factors, such as bioactive peptides, cytokines, leptin and elevated oxidative stress, are involved in the regulation of vascular calcification (6-9).

Several vascular cell types, such as pericytes, vascular smooth muscle cells and fibroblasts, undergo phenotypic change during vascular calcification (10). Vascular smooth muscle cells (VSMCs) have been shown to promote the formation of vascular calcifications (11). Several bone markers have been detected in atherosclerotic plaque, including collagen I, osteocalcin, osteopontin, matrix GLA protein and osteoglycin, indicating that VSMCs can be transdifferentiated into osteoblast-like cells (12).

Connective tissue growth factor (CTGF) is a member of the CCN (cef10/cyr61/ccn1, ctgf/ccn2 and nov/ccn3) family, and has been shown to be upregulated in atherosclerotic plaque (13). CTGF is involved in a variety of autocrine or paracrine functions in several cell types, including fibroblasts, vascular endothelial, neuronal, epithelial and vascular smooth muscle cells, as well as cells of supportive skeletal tissue (14). CTGF is considered to contribute to the development of atherosclerotic lesions by increasing VSMC migration, proliferation, adhesion, apoptosis and the secretion of matrix components (15-17). However, the effect of CTGF on vascular calcification and the transdifferentiation of VSMCs has not yet been fully elucidated.

There is increasing evidence suggesting that extracellular signal-regulated kinase (ERK) plays a crucial role in VSMC calcification (18-21). Furthermore, evidence has accumulated indicating that CTGF activates intracellular signaling pathways, including the ERK, c-Jun N-terminal kinase (JNK) and p38 MAPK pathways (21-25).

The present study aimed to investigate the role of CTGF in VSMC calcification and to determine whether the ERK signaling pathway is involved in this process.

\section{Materials and methods}

Methods. All experiments were carried out in accordance with the Guide for the Care and Use of Laboratory Animals published by the United States National Institutes of Health. 
Study approval was granted by the Ethics Committee of the Second Xiangya Hospital of Central South University, Changsha, China.

Reagents. Recombinant human CTGF was purchased from PeproTech (Rocky Hill, NJ, USA). Antibodies for phosphorylated ERK1/2 (p-ERK1/2), phosphorylated p38 (p-p38), phosphorylated JNK (p-JNK), ERK1/2, p38, or JNK and antimouse and anti-rabbit IgG horseradish peroxidase (HRP)conjugated antibodies were purchased from Santa Cruz Biotechnology, Inc. (Waltham, MA, USA). The ERK-specific inhibitor, PD98059, was purchased from Calbiochem Corp. (San Diego, CA, USA). $\beta$-glycerophosphate was purchased from Sigma (St. Louis, MO, USA). Kunming mice were purchased from the Animal Center, the Second Xiangya Hospital of Central South University. To isolate primary VSMCs, the mice received a $150 \mathrm{mg} / \mathrm{kg}$ intraperitoneal dose of pentobarbital sodium prior to euthanasia which was confirmed by the absence of breathing and a heartbeat.

VSMCs culture. Primary VSMCs used in the present study were obtained from mouse thoracic aortas by enzymatic digestion as previously described and identified by immunostaining for smooth muscle specific $\alpha$-actin antibody ( $\alpha$-SMA) (26). Briefly, a fragment of mouse thoracic aorta was stripped of adventitia and intima. VSMCs were obtained from the remaining medial layer. The cells were grown in Dulbecco's modified Eagle's medium (DMEM; high glucose, $4.5 \mathrm{~g} / \mathrm{l}$; Gibco-BRL, Carlsbad, CA, USA) containing 5-10\% fetal bovine serum (FBS; HyClone, Logan, UT, USA). The culture medium were changed once every 2 or 3 days.

In vitro calcification. VSMC calcification was induced by the addition of CTGF to the osteogenic mediaum containing $5-10 \%$ FBS in the presence of $0.25 \mathrm{mM}$ ascorbic acid and $10 \mathrm{mM} \beta$-glycerophosphate for 14 days. In some experiments, the cells were incubated with CTGF $(0,10,20,50,100$ and $200 \mathrm{ng} / \mathrm{ml}$ ) for 14 days with medium changes every 2-3 days.

Quantification of calcium deposition. Measurement of total calcium in the extracellular matrix was performed as previously described (27). The cells were decalcified with $0.60 \mathrm{M} \mathrm{HCl}$ for $24 \mathrm{~h}$. The calcium content was determined by measuring the concentrations of calcium in the $\mathrm{HCl}$ supernatant by atomic absorption spectroscopy. Following decalcification, the cells were washed 3 times with phosphate-buffered solution and solubilized with $0.1 \mathrm{M} \mathrm{NaOH} / 0.1 \%$ sodium dodecyl sulfate. The total protein content was determined using a Bradford protein assay. The calcium content of the cell layer was normalized to the protein content.

Alizarin Red S Staining. The calcifying cells that were characterized by the appearance of multilayer nodules were determined by Alizarin Red S staining as previously described (28). Briefly, the cells were washed with PBS 3 times, and fixed in 95\% ethanol for $10 \mathrm{~min}$ at room temperature. The cells were then washed with distilled water 3 times and exposed to $1.0 \%$ Alizarin Red S/Tris- $\mathrm{HCl}$ solution ( $\mathrm{pH}>8.3$ ) for $30 \mathrm{~min}$ at $37^{\circ} \mathrm{C}$. After staining, the cells were washed with distilled water to eliminate non-specific staining.
Quantitative real-time RT-PCR. The effect of CTGF on the expression of bone-related gene markers in the VSMCs was determined by quantitative real-time RT-PCR using a Roche Molecular LightCycler (Roche Applied Science, Indianapolis, IN, USA). Total RNA was isolated from the cultured VSMCs using TRIzol reagent (Invitrogen, Carslbad, CA, USA) and reverse-transcribed into cDNA according to the recommended protocol provided with the reverse transcription kit (Fermentas, Hanover, MD, USA). PCR were performed using specific primers as follows: osteoprotegerin (OPG) sense, 5'-AGTCCGTGAA GCAGGAGT-3' and antisense, 5'-CCATCTGGACATTTTTT GCAAA-3'; osteocalcin (OC) sense, 5'-CTGACAAAGCCTT CATGTCCAA-3' and antisense, 5'-GCGCCGGAGTCTGTT CACTA-3'; core-binding factor subunit $\alpha 1$ (Cbf $\alpha 1) /$ runt-related transcription factor 2 (Runx2) sense, 5'-CCGGTCTCCTTC CAGGAT-3' and antisense, 5'-GGGAACTGCTGTGGC TTC-3'; alkaline phosphatase (ALP) sense, 5'-GACCTCCTC GGAAGACACTC-3' and antisense, 5'-AGGCCCATTGCC ATACAG-3'; and GAPDH sense, 5'-GGCTGCCCAGAACA TCAT-3' and antisense, 5'-CGGACACATTGGGGGTAG-3'; SYBR-Green-based real-time PCR was performed according to the recommendations of the SYBR-Green kit using SYBR Premix Ex Taq [Takara Biotechnology (Dalian) Co., Ltd., Dalian, China].

Western blot analysis. Western blot analysis was performed to investigate the expression of Runx 2 protein and the activity of the downstream intracellular signaling pathways. VSMC extracts were prepared, and the protein concentration was quantified using a Bradford protein assay. A total of $18 \mu \mathrm{g}$ of protein from each cell layer or $30 \mu \mathrm{l}$ conditioned medium from VSMC cultures were loaded onto a $8.0 \%$ polyacrylamide gel. Proteins were separated by electrophoresis on an SDS-PAGE polyacrylamide gel and then were transferred to a $0.45-\mathrm{mm}$ nitrocellulose membrane. The membrane was incubated at $4^{\circ} \mathrm{C}$ overnight with antibodies for Runx2, p-ERK1/2, ERK1/2, JNK, p-JNK, p38 or p-p38 and then with the appropriate HRP-conjugated secondary antibodies. Immunoreactive proteins were detected using an ECL kit and then exposed to X-ray film.

Statistical analysis. SPSS 13.0 software was used for statistical analyses. The results are expressed as the means \pm SD. One-way analysis of variance was used to compare the experimental with the control groups. All experiments were repeated at least 3 times. A P-value $<0.05$ was considred to indicate a statistically significant difference.

\section{Results}

CTGF induces VSMC calcification. To determine the role of CTGF in vascular calcification, we incubated VSMCs from mouse thoracic aortas in DMEM supplemented with $0.25 \mathrm{mM}$ ascorbic acid and $10 \mathrm{mM} \beta$-glycerophosphate in the presence or absence of CTGF for 14 days, and then the amount of calcification in the cells was determined by Alizarin Red S staining. After 14 days, calcifying nodules appeared in CTGF-treated $(50 \mathrm{ng} / \mathrm{ml})$ VSMCs, but fewer nodules appeared in the controls (Fig. 1A and B). We then measured calcification using $50 \mathrm{ng} / \mathrm{ml} \mathrm{CTGF}$. Likewise, the results showed that 
A

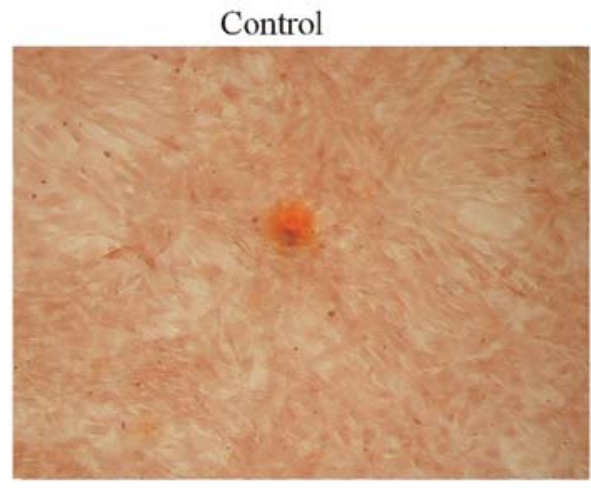

CTGF $50 \mathrm{ng} / \mathrm{ml}$

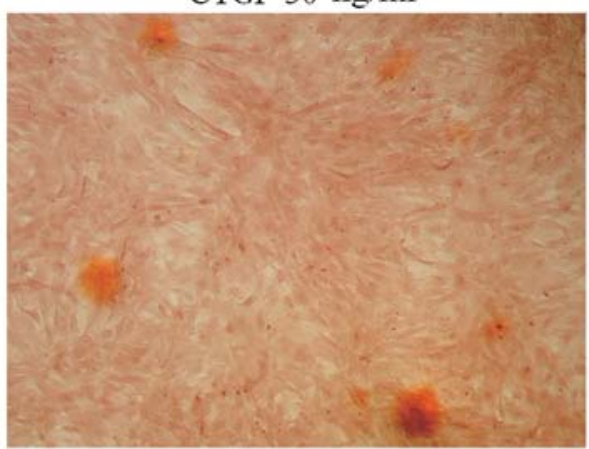

B

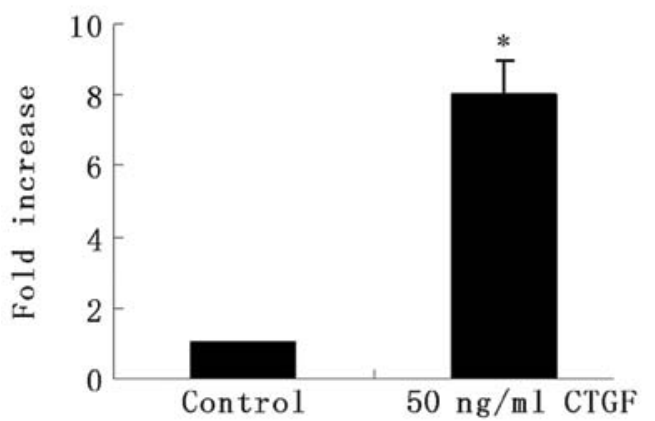

C

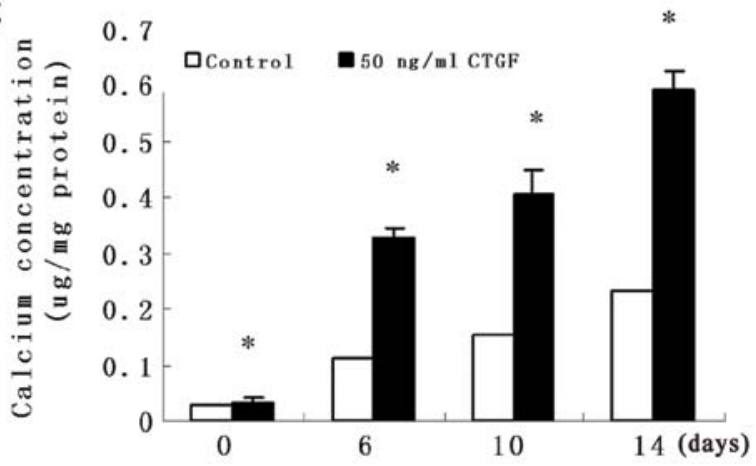

Figure 1. Connective tissue growth factor (CTGF) induces vascular smooth muscle cell (VSMC) calcification. (A) VSMCs were exposed to 50 ng/ml CTGF or the vehicle for 2 weeks with medium change every 2 or 3 days. VSMC calcification was determined by Alizarin Red S staining. Representatives images of microscopic views (x40) from 4 independent experiments are shown. (B) Effect of CTGF on the number of mineralized nodules formed by VSMCs. ("P<0.05 vs. control group, means \pm SD of 3 experiments). (C) Total calcium levels were determined in VSMCs exposed to 50 ng/ml CTGF for $0-14$ days.The data represent the means \pm SD. Results from 3 independent experiments performed in duplicates are shown.

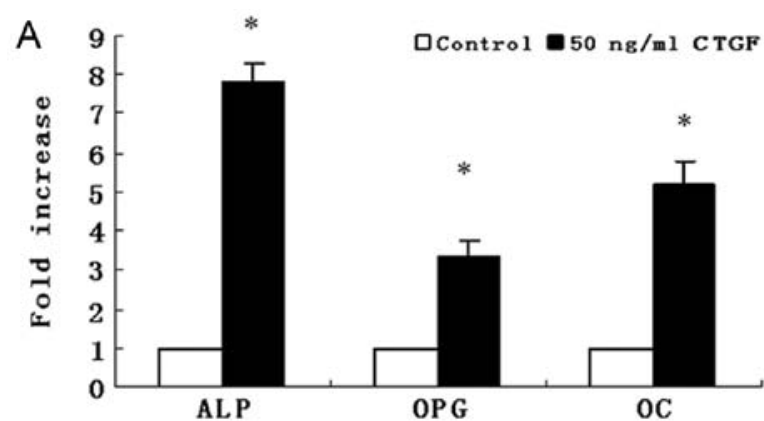

B

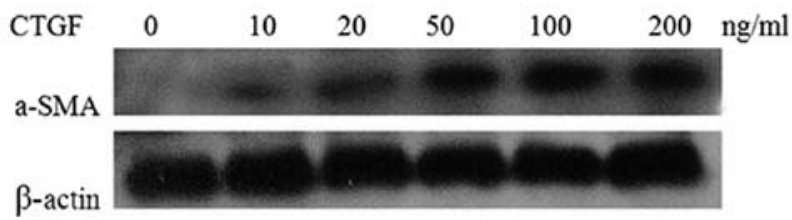

Figure 2. Connective tissue growth factor (CTGF) induces the expression of bone markers and smooth muscle cell markers. (A) Vascular smooth muscle cells (VSMCs) were exposed to $50 \mathrm{ng} / \mathrm{ml}$ CTGF in osteogenic medium for 2 weeks. The expression of the bone-related molecules, alkaline phosphatase (ALP), osteoprotegerin (OPG) and osteocalcin (OC), during VSMC calcification was determined by real-time PCR. Representative results from 3 independent experiments are shown ( $\mathrm{P}<0.05$ compared with the control group; means $\pm \mathrm{SD}$ ). (B) The expression of $\alpha$-SMA protein in VSMCs exposed to $50 \mathrm{ng} / \mathrm{ml} \mathrm{CTGF}$ in osteogenic medium was determined by western blot analysis. Representatives images of western blot analysis are shown.
CTGF promoted the formation of calcium deposits in a timedependent manner (Fig. 1C). These results suggest that CTGF increases vascular calcification.

CTGF induces the expression of bone markers and smooth muscle cell markers. Recent studies have suggested that the process of vascular calcification is similar to the procedure of osteogenesis $(29,30)$. Therefore, we examined the effect of CTGF on osteogenic transdifferentiation by assessing the expression of bone and VSMC markers. The data from the realtime PCR experiments revealed that the mRNA expression of bone markers, such as ALP, OPG and OC was significantly upregulated after the VSMCs were exposed to $50 \mathrm{ng} / \mathrm{ml}$ CTGF for 14 days (Fig. 2A). Western blot analysis was performed to determine the expression of the VSMC marker, $\alpha$-SMA (Fig. 2B). The results revealed that the level of $\alpha$-SMA was also increased during the osteogenic differentiation of VSMCs. These data suggest that CTGF induces the transdifferentiation of VSMCs into osteoblasts. CTGF may promote the proliferation of VSMCs as previously described by Fan et al (15).

CTGF increases Runx2 expression in VSMCs. Runx2, one of the phenotypic markers of osteoblasts, is thought to be a key regulator of osteoblast differentiation and is thought to play an important role in the expression of several bone markers (31). Thus, we examined whether CTGF affects the expression and 

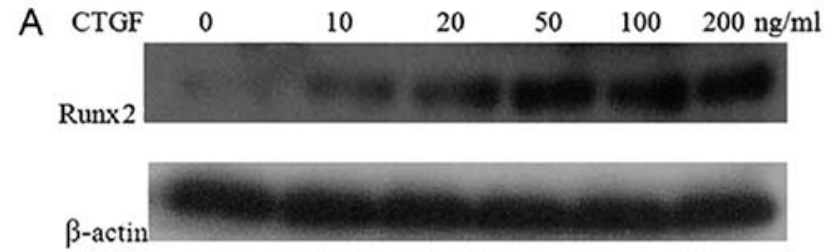

B

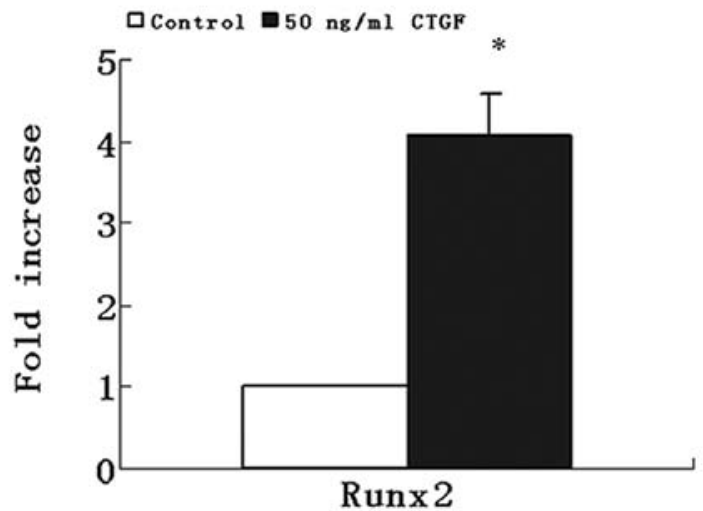

Figure 3. Connective tissue growth factor (CTGF) increases the expression of Runx2 in vascular smooth muscle cells (VSMCs). (A) The expression of Runx2 protein in VSMCs exposed to CTGF $(0,10,20,50,100$ or $200 \mathrm{ng} / \mathrm{ml})$ in osteogenic medium was determined by western blot analysis. (B) Real-time PCR was performed on the VSMCs exposed to $50 \mathrm{ng} / \mathrm{ml} \mathrm{CTGF} \mathrm{in} \mathrm{osteogenic}$ medium for 14 days. The data represent the means \pm SD. Results from 2 independent experiments are shown (" $\mathrm{P}<0.05$ compared with control).

transactivity of Runx 2 during the process of VSMC calcification. Real-time quantitative RT-PCR analysis and western blot analysis were performed to examine the effects of CTGF on the expression of Runx2. The data from western blot analysis showed that the protein expression of Runx 2 was increased in the VSMCs exposed to CTGF $(0,10,20,50,100$ and $200 \mathrm{ng} / \mathrm{ml})$ for 14 days (Fig. 3A) in a concentration-dependent manner. In addition, real-time PCR demonstrated a 4.1-fold increase in Runx2 mRNA expression after the VSMCs were treated with $50 \mathrm{ng} / \mathrm{ml} \mathrm{CTGF}$ for 14 days (Fig. 3B). Thus, these results indicate that CTGF induces the expression of Runx2.

ERK signaling mediates CTGF-induced Runx 2 activation and VSMC calcification. ERK plays an essential role in controlling vascular calcification, and CTGF is involved in the activation of extracellular signaling pathways, including the ERK, JNK and p38 MAPK pathways. Therefore, we determined whether the activation of these signaling pathways by CTGF is required for VSMC calcification. Western blot analysis revealed that CTGF activated ERK and the peak activation of ERK occurred at the 30-min time point (Fig. 4A). However, CTGF had no effect on the phosphorylation of JNK and p38 (Fig. 4A). The effect of the ERK inhibitor (PD98059) on ERK activation was determined by western blot analysis, demonstrating the inhibition of p-ERK in the VSMCs (Fig. 4B). To futher determine whether CTGF stimulates vascular calcification through the ERK pathway, we examined the effect of the ERK signaling pathway inhibitor, PD98059, on VSMC calcification induced by CTGF. Treatment of the VSMCs with the inhibitor of ERK signaling (PD98059) abolished CTGF-induced VSMC calci- fication (Fig. 4C), suggesting that CTGF accelerates VSMC calcification through the ERK signaling pathway. Furthermore, we found that the induction of Runx 2 expression by CTGF was prevented by the ERK inhibitor (Fig. 4D and E). In conclusion, our data indicate that the ERK signaling pathway plays an important role in CTGF-induced VSMC calcification.

\section{Discussion}

CTGF is a $38-\mathrm{kDa}$ cysteine-rich protein that belongs to the $\mathrm{CCN}$ gene family and is known to play an important role in the development of atherosclerosis. CTGF is abundantly expressed in the zone of provisional calcification and in VSMCs around calcification lesions $(13,32)$. CTGF exerts favorable effects on the osteogenic differentiation of mesenchymal stem cells (33). Previous studies have indicated that CTGF promotes atherosclerosis by stimulating VSMC growth, migration, adhesion, apoptosis and the production of extracellular matrix components $(15,17,34)$. However, to our knowledge, there are no data available on the role of CTGF in the osteogenic differentiation of VSMCs. In the present study, we demonstrate that CTGF, at concentrations of 10 to $200 \mathrm{ng} / \mathrm{ml}$, induces the osteogenic differentiation and calcification of VSMCs in a concentrationdependent manner, and that the effect of CTGF on the calcification of VSMCs appears to occur through the activation of the ERK signaling pathway.

Previous studies have demonstrated that vascular calcification is an active and cell-regulated process resembling skeletal mineralization. It is well known that VSMCs can transdifferentiate from contractile into distinct osteoblast-like cells during the process of vascular calcification (35). VSMCs can undergo reversible differentiation into various cells types, including osteoblasts, adipocytes and chondrocytes in atherosclerosis $(36,37)$. Accumulating evidence suggests that the imbalance of phosphate and calcium metabolism, high glucose, chronic inflammation and oxidative stress induce the expression of osteogenic differentiation markers in VSMCs and may contribute to the development of vascular calcification $(38,39)$. We found that CTGF induces the expression of osteocyte phenotype markers and contributes to the phenotypic transition of VSMCs and VSMC calcification.

Bone differentiation markers have been detected in calcified areas, and these proteins are commonly used as markers to indicate the osteogenic/chondrogenic differentiation of VSMCs into osteoblast-like cells (39). These bone-related proteins and genes include transcription factors, such as Gas6, Cbf $\alpha 1$ or Msx2 (40), factors that may contribute to the mineralization process, including ALP, bone sialoprotein and OC, as well as inhibitors of osteochondrogenic mineralization, such as osteopontin, matrix $\gamma$-carboxyglutamic acid and OPG (41). The CTGF-induced VSMC calcification is associated with the induction of bone markers, including ALP, OC and OPG, demonstrating the transdifferentiation of VSMCs into osteoblast-like cells. It has been demonstrated in previous studies that ALP genes are early markers of the transdifferentiation of VSMCs into phenotypically osteoblast-like cells, and that OC proteins are indicators of the late stage of transdifferentiation $(42,43)$. OPG is a key regulator of osteoclastogenesis and may protect cells from arterial calcification (44). Runx2, a key transcription factor for osteoblast and chondrocyte differentia- 
A

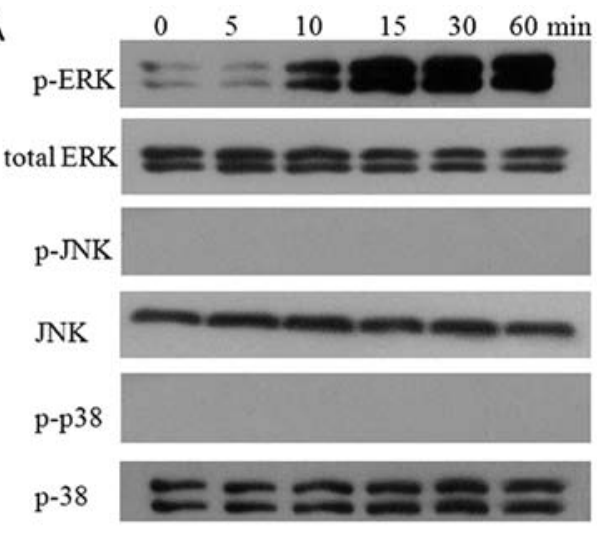

C

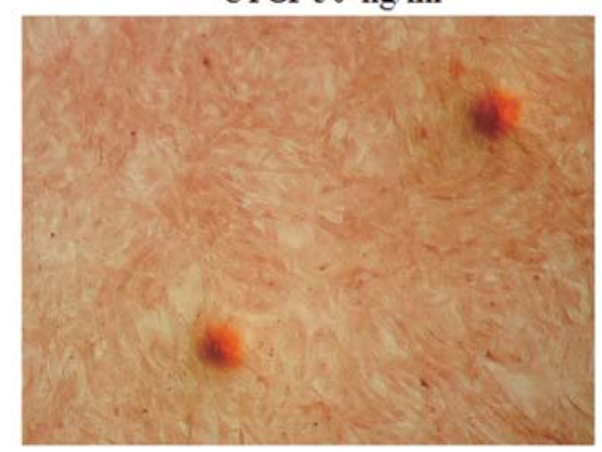

B

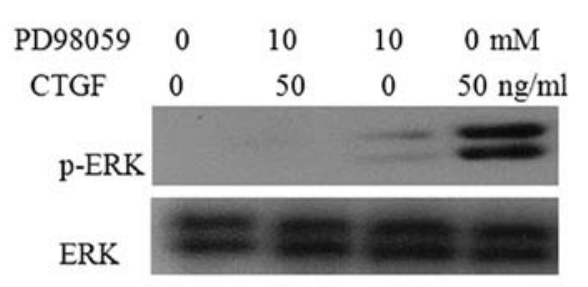

PD98059 + CTGF

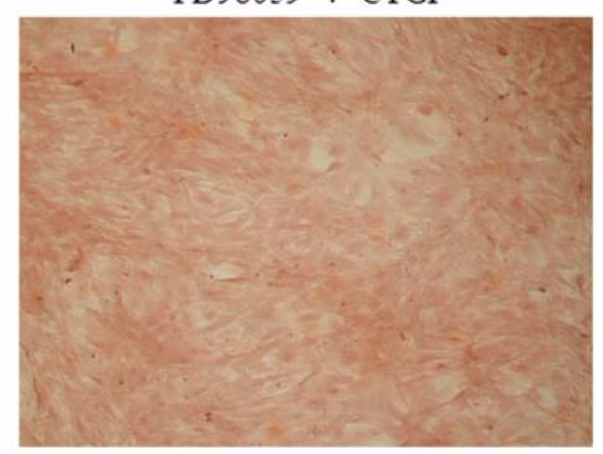

*

D
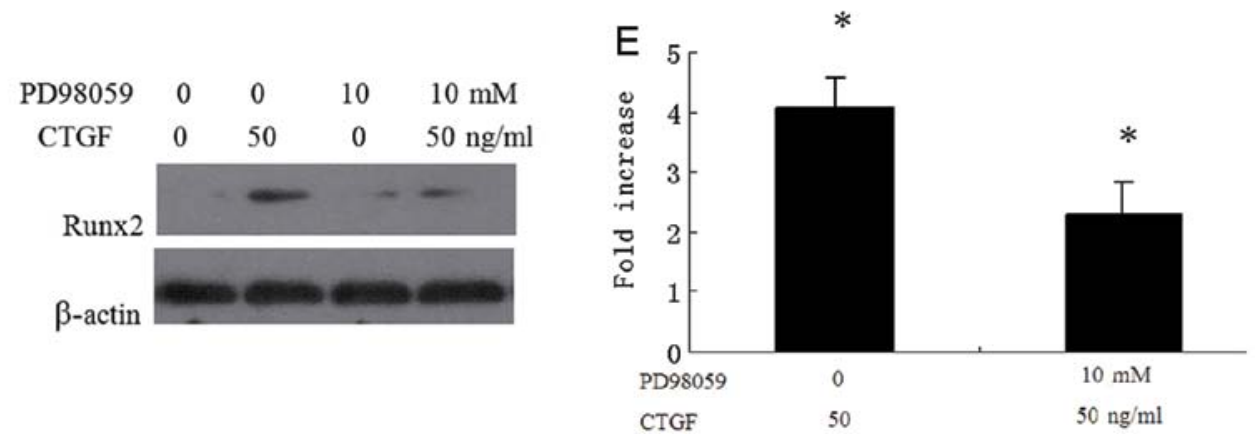

Figure 4. ERK signaling mediates connective tissue growth factor (CTGF)-induced Runx2 activation and vascular smooth muscle cell (VSMC) calcification. (A) The effect of CTGF on the phosphorylation of ERK, JNK and p38 was determined in VSMCs exposed to $50 \mathrm{ng} / \mathrm{ml}$ CTGF in osteogenic medium by western blot analysis. Representative results from 3 independent experiments in duplicate are shown. (B) The effect of inhibitors on the phosphorylation of ERK was determined by western blot analysis of VSMCs pre-treated with PD98059 $(25 \mu \mathrm{M})$ for $30 \mathrm{~min}$ and then exposed to $50 \mathrm{ng} / \mathrm{ml} \mathrm{CTGF}$ for $15 \mathrm{~min}$ in osteogenic medium. (C) The effect of ERK inhibitor (PD98059) on the CTGF-induced VSMC calcification was determined by Alizarin Red S Staining. VSMCs were pre-treated with PD98059 $(25 \mu \mathrm{M})$ for $30 \mathrm{~min}$ and then exposed to $50 \mathrm{ng} / \mathrm{ml}$ CTGF in osteogenic medium for 14 days. The effect of ERK inhibitor on the expression of Runx2 was determined by (D) western blot analysis and (E) real-time PCR. VSMCs were pre-treated with ERK inhibitor (PD98059) for 30 min and then exposed to $50 \mathrm{ng} / \mathrm{ml} \mathrm{CTGF}$ in osteogenic medium for 2 weeks. Treatment was repeated every 3 days for 2 weeks. The data represent the means \pm SD. Representative western blot analysis and real-time PCR results from 2 independent experiments are shown.

tion, regulates the phenotypic conversion of VSMCs. Previous studies have indicated that Runx2 plays an essential role in oxidative stress-induced VSMC calcification in vitro, and Runx2 alone is sufficient to induce VSMC calcification (38). In this study, we found that CTGF promoted the phenotypic switch of VSMCs, with the CTGF-treated VSMCs showing significant increases in ALP, OC, OPG and Runx2 expression. CTGF induced VSMC calcification with the induction of bone markers; however, it is interesting that CTGF is unlike other factors, such as oxidative stress and fibronectin that can downregulate the expression of the VSMC-specific marker, $\alpha$-SMA $(18,38)$. Perhaps CTGF can not only induce the osteogenic differentiation of VSMCs but can also promote the growth of VSMCs, both of which contribute to vascular calcification.

We further determined the molecular signals involved in CTGF-induced VSMC calcification. A number of studies have demonstrated that the ERK pathway is involved in vascular calcification with the ERK pathway activated by oxidized low-density lipoprotein (LDL), PI3-kinase, homocysteine, fibronectin and fibroblast growth factor-2 (18,19,45-47). p38 MAPK and JNK also contribute to the induction of vascular calcification. Advanced glycation end products induce the calcification of VSMCs via the p38 MAPK signaling pathway (48). In this study, we found that the CTGF-activated ERK signaling, but not p38 MAPK or JNK signaling, was 
required for the process of vascular calcification. Furthermore, PD98059, an ERK-specific inhibitor, significantly suppressed the effect of CTGF on VSMC calcification and osteoblastic expression. These findings suggest that CTGF enhances vascular calcification and the osteogenic differentiation of VSMCs through the ERK signaling pathway.

Vascular calcification correlates with osteoporosis, and the vascular RANKL system is a common mechanism of osteoporosis and vascular calcification. A recent study found that estrogen inhibits vascular calcification via the vascular RANKL system (49). Our previous study showed that estrogen inhibits the expression of CTGF in a dosedependent manner (50). It is not known whether the effect of estrogen on vascular calcification is associated with the effect of CTGF on VSMC calcification and osteoblast differentiation, and whether estrogen inhibits vascular calcification by inhibiting the expression of CTGF. Therefore, further studies are warranted to explore the combined effect of CTGF and estrogen on VSMC calcification and osteoblast differentiation.

Taken together, our results suggest that CTGF enhances in vitro calcification by inducing the osteogenic differentiation and calcification of VSMCs, and that this effect may be occur through the activation of the ERK pathway. The ERK-specific inhibitor, PD98059, suppressed nodule formation, calcium deposition and the expression of bone markers. Our findings reveal the function of CTGF in vascular calcification and may aid in the development of novel therapies for the prevention and treatment of diseases associated with vascular calcification.

\section{Acknowledgements}

This study was supported by the China National Natural Scientific Foundation (grant nos. 30600661, 30572078 and 81070246). We thank Zhongjian Xie, Rongrong Cui and Qiuhua Liang for their assistance and advice.

\section{References}

1. London GM, Marchais SJ, Guerin AP and Metivier F: Arteriosclerosis, vascular calcifications and cardiovascular disease in uremia. Curr Opin Nephrol Hypertens 14: 525-531, 2005.

2. Mackenzie NC, Zhu D, Longley L, Patterson CS, Kommareddy S and MacRae VE: MOVAS-1 cell line: A new in vitro model of vascular calcification. Int J Mol Med 27: 663-668, 2011.

3. Mikhaylova L, Malmquist J and Nurminskaya M: Regulation of in vitro vascular calcification by BMP4, VEGF and Wnt3a. Calcif Tissue Int 81: 372-381, 2007.

4. Otto CM, Kuusisto J, Reichenbach DD, Gown AM and O'Brien KD: Characterization of the early lesion of 'degenerative' valvular aortic stenosis. Histological and immunohistochemical studies. Circulation 90: 844-853, 1994.

5. Watson KE, Bostrom K, Ravindranath R, Lam T, Norton B and Demer LL: TGF-beta 1 and 25-hydroxycholesterol stimulate osteoblast-like vascular cells to calcify. J Clin Invest 93: 2106-2113, 1994

6. Kurabayashi M: Differentiation of smooth muscle cells and vascular calcification. Clin Calcium 20: 1637-1644, 2010 (In Japanese).

7. Nakagami H, Osako MK and Morishita R: New concept of vascular calcification and metabolism. Curr Vasc Pharmacol 9: 124-127, 2011.

8. Parhami F, Morrow AD, Balucan J, et al: Lipid oxidation products have opposite effects on calcifying vascular cell and bone cell differentiation. A possible explanation for the paradox of arterial calcification in osteoporotic patients. Arterioscler Thromb Vasc Biol 17: 680-687, 1997.
9. Shioi A, Katagi M, Okuno Y, et al: Induction of bone-type alkaline phosphatase in human vascular smooth muscle cells: roles of tumor necrosis factor-alpha and oncostatin $\mathrm{M}$ derived from macrophages. Circ Res 91: 9-16, 2002.

10. Duan XY, Xie PL, Ma YL and Tang SY: Omentin inhibits osteoblastic differentiation of calcifying vascular smooth muscle cells through the PI3K/Akt pathway. Amino Acids 41: 1223-1231, 2011.

11. Iyemere VP, Proudfoot D, Weissberg PL and Shanahan CM: Vascular smooth muscle cell phenotypic plasticity and the regulation of vascular calcification. J Intern Med 260: 192-210, 2006.

12. Yamaguchi A: Vascular calcification and bone-related factors Clin Calcium 12: 1078-1083, 2002 (In Japanese).

13. Oemar BS, Werner A, Garnier JM, et al: Human connective tissue growth factor is expressed in advanced atherosclerotic lesions. Circulation 95: 831-839, 1997.

14. Moussad EE and Brigstock DR: Connective tissue growth factor: what's in a name. Mol Genet Metab 71: 276-292, 2000.

15. Fan WH, Pech M and Karnovsky MJ: Connective tissue growth factor (CTGF) stimulates vascular smooth muscle cell growth and migration in vitro. Eur J Cell Biol 79: 915-923, 2000.

16. Hishikawa K, Oemar BS, Tanner FC, Nakaki T, Fujii T and Luscher TF: Overexpression of connective tissue growth factor gene induces apoptosis in human aortic smooth muscle cells. Circulation 100: 2108-2112, 1999.

17. Kanazawa S, Miyake T, Kakinuma T, Tanemoto K, Tsunoda T and Kikuchi K: The expression of platelet-derived growth factor and connective tissue growth factor in different types of abdominal aortic aneurysms. J Cardiovasc Surg (Torino) 46: 271-278, 2005.

18. Ding HT, Wang CG, Zhang TL and Wang K: Fibronectin enhances in vitro vascular calcification by promoting osteoblastic differentiation of vascular smooth muscle cells via ERK pathway. J Cell Biochem 99: 1343-1352, 2006.

19. Li J, Chai S, Tang C and Du J: Homocysteine potentiates calcification of cultured rat aortic smooth muscle cells. Life Sci 74: 451-461, 2003

20. Liao XB, Zhou XM, Li JM, et al: Taurine inhibits osteoblastic differentiation of vascular smooth muscle cells via the ERK pathway. Amino Acids 34: 525-530, 2008.

21. Yang M, Huang H, Li J, Li D and Wang H: Tyrosine phosphorylation of the LDL receptor-related protein (LRP) and activation of the ERK pathway are required for connective tissue growth factor to potentiate myofibroblast differentiation. FASEB J 18: 1920-1921, 2004

22. Huang HC, Yang M, Li JZ and Wang HY: Connective tissue growth factor promotes the proliferation of myofibroblast through Erk-1/2 signaling pathway. Zhonghua Yi Xue Za Zhi 85: 1322-1326, 2005 (In Chinese).

23. Johnson PR, Burgess JK, Ge Q, et al: Connective tissue growth factor induces extracellular matrix in asthmatic airway smooth muscle. Am J Respir Crit Care Med 173: 32-41, 2006.

24. Ponticos M, Holmes AM, Shi-wen X, et al: Pivotal role of connective tissue growth factor in lung fibrosis: MAPK-dependent transcriptional activation of type I collagen. Arthritis Rheum 60: 2142-2155, 2009

25. Yosimichi $G$, Nakanishi $T$, Nishida $T$, Hattori $T$, TakanoYamamoto T and Takigawa M: CTGF/Hcs24 induces chondrocyte differentiation through a p38 mitogen-activated protein kinase (p38MAPK), and proliferation through a p44/42MAPK/ extracellular-signal regulated kinase (ERK). Eur J Biochem 268: 6058-6065, 2001.

26. Ross R, Glomset J, Kariya B and Harker L: A platelet-dependent serum factor that stimulates the proliferation of arterial smooth muscle cells in vitro. Proc Natl Acad Sci USA 71: 1207-1210, 1974.

27. Wada T, McKee MD, Steitz S and Giachelli CM: Calcification of vascular smooth muscle cell cultures: inhibition by osteopontin. Circ Res 84: 166-178, 1999.

28. Alexander MY, Wilkinson FL, Kirton JP, et al: Identification and characterization of vascular calcification-associated factor, a novel gene upregulated during vascular calcification in vitro and in vivo. Arterioscler Thromb Vasc Biol 25: 1851-1857, 2005.

29. Shioi A: Molecular mechanisms of vascular calcification. Clin Calcium 20: 1611-1619, 2010 (In Japanese).

30. Sinha S, Eddington $H$ and Kalra PA: Vascular calcification: lessons from scientific models. J Ren Care 35 (Suppl 1): 51-56, 2009. 
31. Nakano-Kurimoto R, Ikeda K, Uraoka M, et al: Replicative senescence of vascular smooth muscle cells enhances the calcification through initiating the osteoblastic transition. Am J Physiol Heart Circ Physiol 297: H1673-H1684, 2009.

32. Yamaai T, Nakanishi T, Asano M, et al: Gene expression of connective tissue growth factor (CTGF/CCN2) in calcifying tissues of normal and cbfa1-null mutant mice in late stage of embryonic development. J Bone Miner Metab 23: 280-288, 2005.

33. Wang JJ, Ye F, Cheng LJ, et al: Osteogenic differentiation of mesenchymal stem cells promoted by overexpression of connective tissue growth factor. J Zhejiang Univ Sci B 10: 355-367, 2009.

34. Game BA, He L, Jarido V, et al: Pioglitazone inhibits connective tissue growth factor expression in advanced atherosclerotic plaques in low-density lipoprotein receptor-deficient mice. Atherosclerosis 192: 85-91, 2007.

35. Steitz SA, Speer MY, Curinga G, et al: Smooth muscle cell phenotypic transition associated with calcification: upregulation of Cbfal and downregulation of smooth muscle lineage markers. Circ Res 89: 1147-1154, 2001.

36. Johnson RC, Leopold JA and Loscalzo J: Vascular calcification: pathobiological mechanisms and clinical implications. Circ Res 99: 1044-1059, 2006.

37. Tyson KL, Reynolds JL, McNair R, Zhang Q, Weissberg PL and Shanahan CM: Osteo/chondrocytic transcription factors and their target genes exhibit distinct patterns of expression in human arterial calcification. Arterioscler Thromb Vasc Biol 23 : 489-494, 2003.

38. Byon CH, Javed A, Dai Q, et al: Oxidative stress induces vascular calcification through modulation of the osteogenic transcription factor Runx2 by AKT signaling. J Biol Chem 283: 15319-15327, 2008.

39. Liu Y and Shanahan CM: Signalling pathways and vascular calcification. Front Biosci 16: 1302-1314, 2011.

40. Son BK and Akishita M: Mechanism of vascular calcification. Clin Calcium 17: 319-324, 2007 (In Japanese).

41. Valdivielso JM: Vascular calcification: types and mechanisms. Nefrologia 31: 142-147, 2011 (In Spanish).
42. Gallop PM, Lian JB and Hauschka PV: Carboxylated calciumbinding proteins and vitamin K. N Engl J Med 302: 1460-1466, 1980.

43. Stinson RA and Hamilton BA: Human liver plasma membranes contain an enzyme activity that removes membrane anchor from alkaline phosphatase and converts it to a plasma-like form. Clin Biochem 27: 49-55, 1994.

44. Zhang J, Fu M, Myles D, et al: PDGF induces osteoprotegerin expression in vascular smooth muscle cells by multiple signal pathways. FEBS Lett 521: 180-184, 2002.

45. Bear M, Butcher M and Shaughnessy SG: Oxidized low-density lipoprotein acts synergistically with beta-glycerophosphate to induce osteoblast differentiation in primary cultures of vascular smooth muscle cells. J Cell Biochem 105: 185-193, 2008.

46. Nakahara T, Sato H, Shimizu T, et al: Fibroblast growth factor-2 induces osteogenic differentiation through a Runx2 activation in vascular smooth muscle cells. Biochem Biophys Res Commun 394: 243-248, 2010

47. You $\mathrm{H}$, Yang $\mathrm{H}$, Zhu Q, et al: Advanced oxidation protein products induce vascular calcification by promoting osteoblastic trans-differentiation of smooth muscle cells via oxidative stress and ERK pathway. Ren Fail 31: 313-319, 2009.

48. Tanikawa T, Okada Y, Tanikawa R and Tanaka Y: Advanced glycation end products induce calcification of vascular smooth muscle cells through RAGE/p38 MAPK. J Vasc Res 46: 572-580, 2009.

49. Osako MK, Nakagami H, Koibuchi N, et al: Estrogen inhibits vascular calcification via vascular RANKL system: common mechanism of osteoporosis and vascular calcification. Circ Res 107: 466-475, 2010.

50. Peng $\mathrm{Y}$, Wu $\mathrm{M}$, Huang $\mathrm{J}$, Xie $\mathrm{H}$, Meng $\mathrm{J}$ and Liao E: $17-\beta$ estradiol down-regulates the expression of CTGF in vascular smooth muscle cells of mouse. The 10th National Endocrinology Conference of Chinese Medical Association, Suzhou, Jiangsu Province, P.R. China, 2011 (In Chinese). 